\title{
Thermographic evaluation of Er-doped silica fiber as IR-to- visible image converter
}

\author{
Gonzalo Paez ${ }^{1}$ and M. K. Scholl ${ }^{2}$
}

${ }^{1}$ Centro de Investigaciones en Optica, León, Guanajuato, México

${ }^{2}$ Alenka Associates, Tempe, Arizona, USA

\begin{abstract}
A novel concept for IR-to-visible converter is based on the up-conversion, upon pumping with the near IR radiation. The theoretical heat transfer predictions were followed by the experimental results whereby the fiber was maintained at a series of temperatures. Currently, an IR camera (2003 CEDIP), sensitive in the spectral range from $8 \mu \mathrm{m}$ to $12 \mu \mathrm{m}$ is being used to ascertain the validity of the temperature measurements.
\end{abstract}

\section{Upconversion-based IR-to-visible converter}

Recently, temperature sensor concept has been described that is based on the upconversion process in the Er-doped silica fiber. When the material is irradiated with the near IR radiation of about $800 \mathrm{~nm}$, the visible radiation in the spectral interval $400 \mathrm{~nm}$ to $500 \mathrm{~nm}$ is generated, with the spectral distribution depending only on the sample temperature.[1,2] This process may be described as the conversion of spatial temperature distribution to the spatial distribution of visible radiation - a visible image.[3]

For feasibility assessment, we are using an optical fiber. The first step is to quantify its capacity to generate, maintain, and refresh the thermal image in space and time. For the spatial resolution determination, two hot spots are imaged on a piece of the Er-doped silica in a form of a fiber. The fiber photograph is shown in Figure 1: part (a) is in the visible and (b) is in the IR, also illustrating temperature distribution. An important feature of a dynamic IR image converter is its ability to conserve and erase the thermal image, once it has been imprinted on its surface. Temperature distributions are generated with several types of irradiation sources.

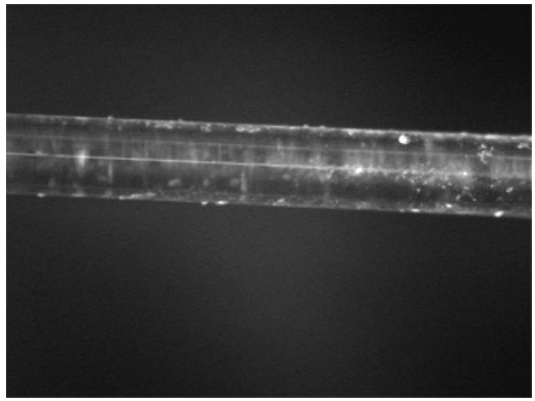

Fig. 1a. The green fluorescence generated in the fiber core, upon pumping with the near IR
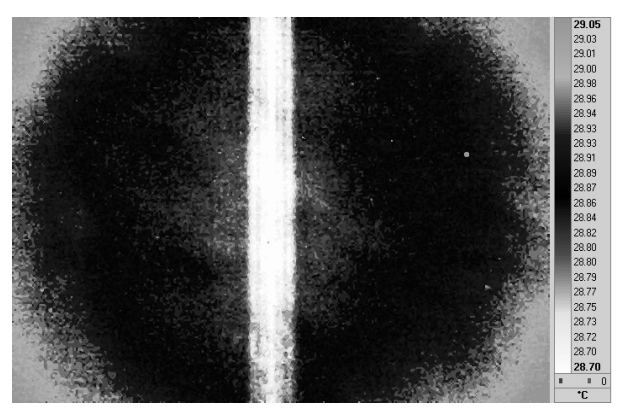

Fig. 1b. Fiber observed in IR, with core temperature distribution from $28.70 \mathrm{C}$ to 28.77 C. (White is cooler.) 


\section{Experimental}

We evaluated the fiber temperature distribution using the IR thermometry. We measured the response of the fiber to thermal stimulation, in the static and dynamic sense. We took images of the fiber shown in Figure 1a (horizontal) with the outside diameter of only $90 \mu \mathrm{m}$. The camera field of view is adjusted to $760 \mu \mathrm{m}$ by $1010 \mu \mathrm{m}$. Thus, the fiber length (oriented vertically in all thermograms) corresponds to $760 \mu \mathrm{m}$. On all the subsequent thermograms, the field of view is only $760 \mu \mathrm{m}$ by $640 \mu \mathrm{m}$.

The digital thermographs were recorded at 60 frames per second on with 14 bits depth for 10 seconds. The measurements include spatial response in $x$ - and $y$ direction (transverse and longitudinal) taken as a single image. The temporal information is recorded in the sequential frames. The measurement procedure and technique presented for the fiber may be similarly used for the evaluation of thermal phenomena in optical waveguides and self-heating in fiber lasers.

The IR camera (2003 CEDIP),, sensitive from $8 \mu \mathrm{m}$ to $14 \mu \mathrm{m}$, is capable of detecting temperature differences of $0.01 \mathrm{~K}(\mathrm{NEDT}=0.01 \mathrm{~K})$. The temperature range is shown on the scale on the right, corresponding to the false color distribution indicated for every frame.[4] For the case of that in Fig. 1b, it is from $28.7 \mathrm{C}$ to 29.05 C. The room temperature is $25 \mathrm{C}$, and the fiber is at a nearly uniform temperature of from $28.7 \mathrm{C}$ to $28.8 \mathrm{C}$, within the accuracy of the camera NEDT.

\subsection{Thermal Point Source}

The fiber response to thermal point source at the center is determined by placing a hot, polished, low-emissivity copper wire running at 90 degrees against the back of the fiber. The latter is vertical and the green wire is horizontal in Figure 2a. Figure $2 \mathrm{~b}$ shows the distribution of temperature as a function of two perpendicular coordinates.

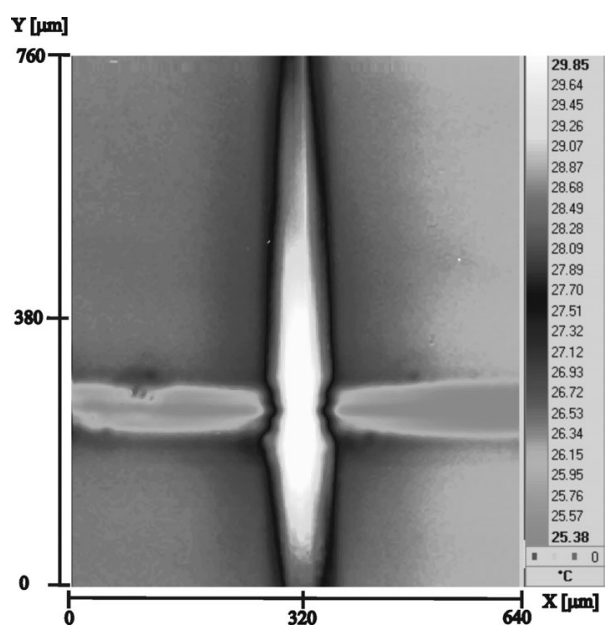

(a) thermogram with false color

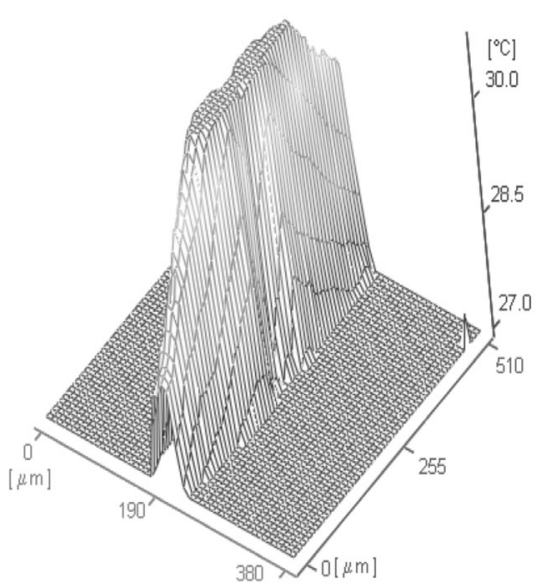

(b) two-dimensional temperature distribution in the fiber

Fig. 2. Steady state temperature in the fiber in contact with a hot wire at one point:

(a) thermogram with false color, and (b) two-dimensional temperature distribution. 
The temperature of the fiber in contact with the hot wire at steady state is confirmed by the thermocouple, with the resolution $0.1 \mathrm{C}$. Pixel temperature decreases with the distance from the hot spot. This result demonstrates the response of the pixel element of the IR to visible transducer to the spatial impulse in the steady state.

The data in Figure 2 isused to obtain temperature along the fiber (Figure 3a) and across it (Figure $3 b$ ).

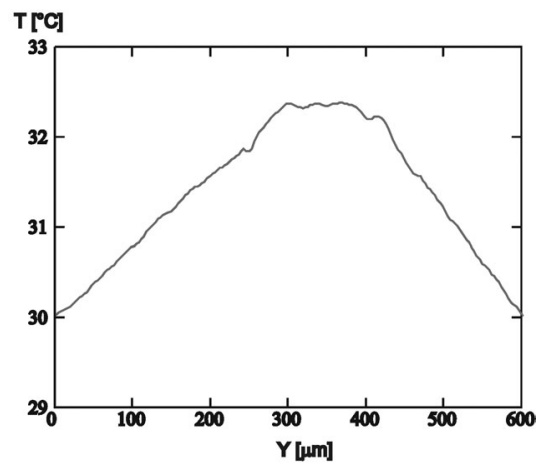

Fig. 3a. Temperature profile along the fiber length illustrates that the point in contact is hottest.

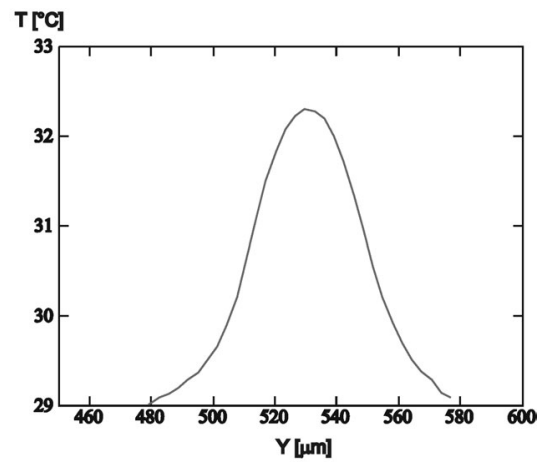

Fig. 3b. Temperature profile across the fiber at the point in contact with hot wire is asymmetrical.

\section{$\underline{2.2 \text { Temporal response }}$}

We consider two cases of dynamic response to point input. First, the constant thermal source is turned on at $t=1.21 \mathrm{sec}$ to a fiber at thermal equilibrium at about $30.3 \mathrm{C}$. From the sequence of frames, we obtain the graph of temperature of the heated pixel as a function of time, presented in Figure $4 a$. We calculate the turn-on time constant of $0.2 \mathrm{sec}$ for the fiber temperature propagation.

We repeat this experiment for the case of constant heat input that is turned off at time $\mathrm{t}=0.7 \mathrm{sec}$, with the temperature vs time results exhibited in Figure $4 \mathrm{~b}$.

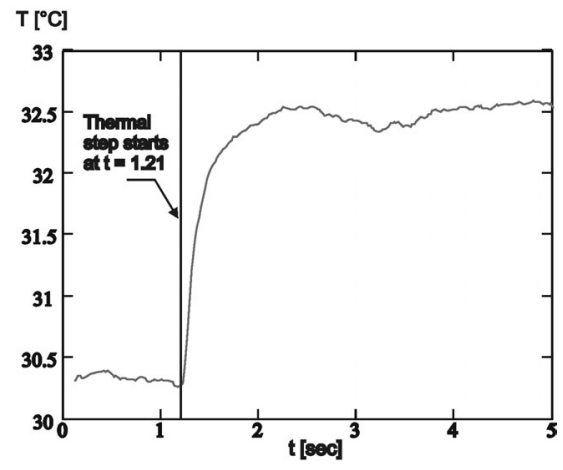

Fig. 4a. Temperature response of the pixel to the constant heat input at time $\mathrm{t}=1.21 \mathrm{sec}$.

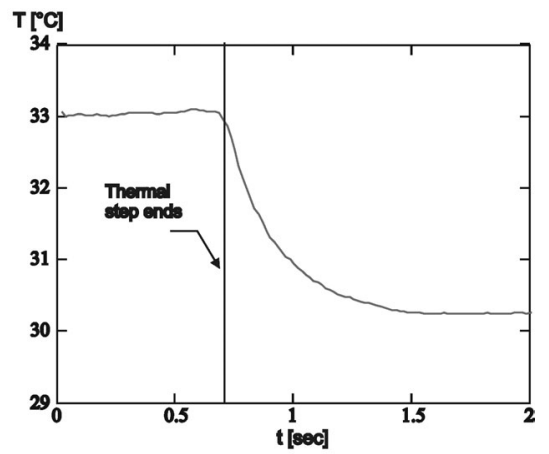

Fig. 4b. Temperature response of the heated pixel to the sudden turn-off of heat source at time $t=0.7 \mathrm{sec}$. 
This time we find that the time constant on turn-off is $0.22 \mathrm{sec}$, slightly larger than the one obtained upon heating. This is to be expected, considering that the fiber is cooled only by convection. Figure 5 shows the temperature evolution along the fiber after the constant heat source has been turned on.

\subsection{Theory}

The feasibility of the converter concept may be demonstrated by determining the temporal and the spatial resolution of this device upon imaging a set of two hot spots on its surface. Thus, the development of the thermal model is reported here to describe the time dependent heat transfer within the fiber. Upon application of this model, different materials may be evaluated as to their feasibility and to predict their potential performance as the radiation converters. In the evaluation of the model performance, the Er-doped silica fiber is reported here.

A general 3D-, time dependent heat equation with localized, surface sources and sinks has been developed previously.[4-7] Due to the geometry of the fiber, the cylindrical coordinates are applied appropriately.

$$
c_{e} \rho \frac{\partial T(r, \phi, z, t)}{\partial t}=\nabla[\bar{k} \cdot \nabla T(r, \phi, z, t)]-p_{s}(R, \phi, z, t)
$$

The symbols are defined as follows: $\mathrm{T}(\rho, \phi, z, \mathrm{t})$ is the temperature distribution within the fiber, $[\mathrm{K}]$; $\mathrm{t}$ is time, $[\mathrm{s}] ;(\rho, \phi, z)$ are the cylindrical coordinates; is density, $\left[\mathrm{kg} / \mathrm{m}^{3}\right] ; \mathrm{c}_{\mathrm{e}}$ is the specific heat capacity, $[\mathrm{J} /(\mathrm{kg} \mathrm{K})] ; \mathbf{k}$ is the heat conductivity vector $\left(k_{x}, k_{y}, k_{z}\right),[W /(m K)] ; p_{s}(R, \phi, z, t)$ is the absorbed power per unit area on the fiber surface (power area density), and it depends on position and time, $\left[\mathrm{W} / \mathrm{m}^{2}\right]$; and $\mathrm{R}$ is the fiber radius, $[\mathrm{m}]$.

Figure 6 displays the calculated increase in temperature as a function of the fiber length, with time as a parameter. Constant power is absorbed from time $t=$ 0 to $t=18 \mathrm{~ms}$ at two pixels, in difference from the measured results. Theoretical pixels of micrometer size may be easily heated in computer simulations. In the future work, the experimental results and computer simulations will be compared.

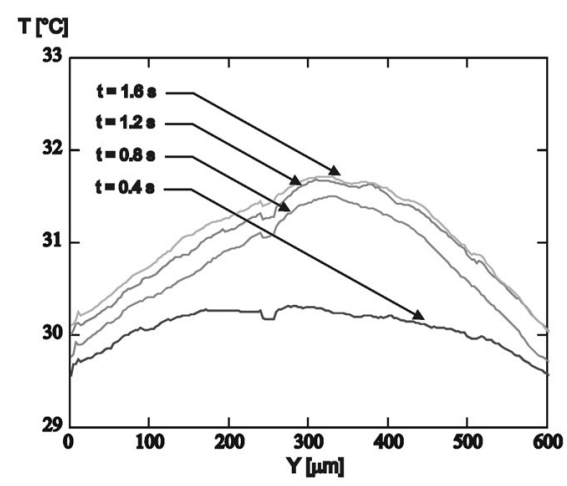

Fig. 5. Temperature distribution along the fiber (y-direction) for different observation times, after the constant heat input has been turned on the central pixel.

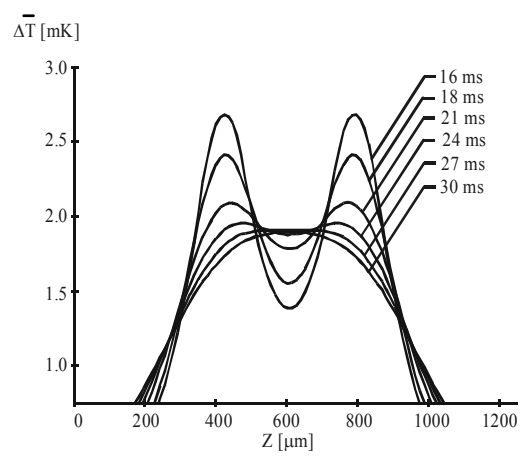

Fig. 6. Calculated increase in temperature as a function of the fiber length, with time as a parameter. Constant power is absorbed from time $\mathrm{t}=0$ to $\mathrm{t}=18 \mathrm{msec}$. 


\section{IR-to-visible converter concept}

Experimental setup to demonstrate the feasibility of the Er-doped silica fiber to function as the IR-to-VIS transducer is illustrated in Figure 7. The arrow on the left represents the IR object that we try to render visible. An IR lens is used to project this object onto the fiber, shown on the right. The IR radiation passes through a 45-degree inclined dichroic beam splitter with a transmission transition wavelength at about $800 \mathrm{~nm}$. When the radiation is incident and absorbed by the fiber, the Er-ions (upon pumping with the near IR radiation) emit the visible radiation in the $500-600 \mathrm{~nm}$ range. Inside the converter fibers, this IR image produces an incremental temperature increase, a latent IR image. The Er-ions in the doped silica fiber, upon pumping with the near IR radiation, produce visible radiation in proportion to the magnitude of the temperature increment. This visible radiation is emitted isotropically, and therefore, also in the transverse direction. The lens of the low-light-level high resolution CCD camera collects it.

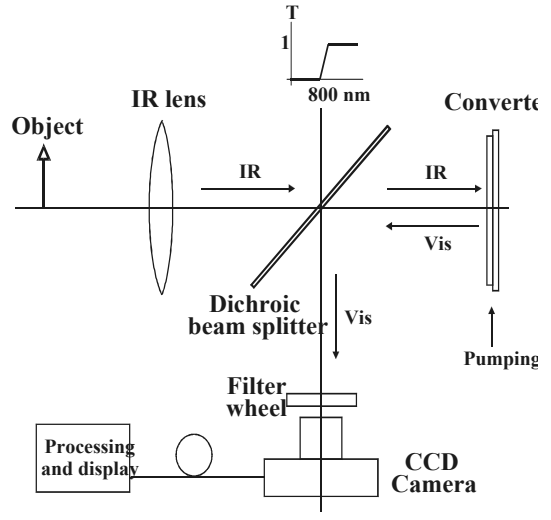

(a) schematic arrangement

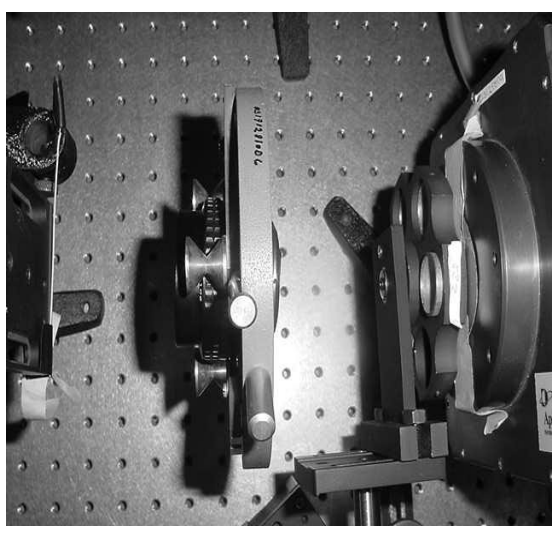

(b) photo of principal components

Fig. 7. Experimental layout of the optical components in the proposed IR-tovisible converter: (a) schematic arrangement, and (b) photograph of principal components.

\section{Summary}

We proposed to use the rare-earth-doped silica as the IR-to-visible converter. After the thermal image has been generated, the erbium-doped silica (upon suitable pumping) up-converts the IR radiation into the visible, in proportion to the local temperature increase. Thus, the incident IR radiation is first absorbed; then it is converted into heat that results in the local temperature increase. This, in turn, causes the emission of the visible radiation, upon pumping. The visible radiation is easily observed with a high-resolution (visible) CCD camera.

Previously, time-dependent, 3-D heat transfer model for the fiber geometry, employing the finite element method, was developed. In this report, we used the IR thermography to evaluate the temperature distribution in the fiber upon application of constant thermal source or upon turning off the constant 
thermal source. Furthermore, we evaluated quantitatively the twodimensional temperature distribution on the fiber. Two separated pixels cannot be accomplished experimentally at this time, due to their small size. The preliminary experimental results of the study on the Er-doped silica demonstrate the feasibility of this transducer.

\section{REFERENCES}

1. Sandoval, J., Paez, G., Strojnik, M. "Er-doped Silica dynamic IR-tovisible image converter," Inf. Phys. \& Tech., 45 (2004).

2. Lopez, V., Paez, G., Strojnik, M. "Sensitivity of a temperature sensor, employing ratio of fluorescence power in a band," Inf. Phys. \& Tech., 45 (2004).

3. Sandoval, J., G. Paez, and M. Strojnik, Paez, G., Scholl, M. S. "Feasibility evaluation of IR-to-Visible Converter," Opt. Eng.. December 2003.Scholl, M. S. "Spatial and temporal effects due to target irradiation: A Study," Appl. Opt., 21 (9), 1615-1620 (1982).

4. Scholl, M. S. "Errors in radiance simulation and scene discrimination," Appl. Opt., 21 (10), 1839-1843 (1982).

5. Scholl, M. S. "Thermal considerations in the design of a dynamic IR source," Appl. Opt., 21 (4), 660-667 (1982).

6. Scholl, M. S. "Target temperature distribution generated and maintained by a scanning laser beam," Appl. Opt., 21 (12), 2146-2152 (1982).

7. Scholl, M. S. "Spatial and Temporal Effects due to Target Irradiation: A Study," Appl. Opt., 21 (9), 1615-1620 (1982). 